\title{
Molecular characterization of a mariner-like element in the Atta sexdens rubropilosa genome
}

\author{
P. Rezende-Teixeira, J.B. do Amaral, F. Siviero and G.M. Machado-Santelli \\ Departamento de Biologia Celular e do Desenvolvimento, \\ Instituto de Ciências Biomédicas, Universidade de São Paulo, São Paulo, SP, Brasil \\ Corresponding author: P. Rezende-Teixeira \\ E-mail: paularez@usp.br \\ Genet. Mol. Res. 11 (2): 1475-1485 (2012) \\ Received March 28, 2011 \\ Accepted August 1, 2011 \\ Published May 21, 2012 \\ DOI http://dx.doi.org/10.4238/2012.May.21.4
}

\begin{abstract}
Mobile elements are widely present in eukaryotic genomes. They are repeated DNA segments that are able to move from one locus to another within the genome. They are divided into two main categories, depending on their mechanism of transposition, involving RNA (class I) or DNA (class II) molecules. The mariner-like elements are class II transposons. They encode their own transposase, which is necessary and sufficient for transposition in the absence of host factors. They are flanked by a short inverted terminal repeat and a TA dinucleotide target site, which is duplicated upon insertion. The transposase consists of two domains, an N-terminal inverted terminal repeat binding domain and a $\mathrm{C}$-terminal catalytic domain. We identified a transposable element with molecular characteristics of a mariner-like element in Atta sexdens rubropilosa genome. Identification started from a PCR with degenerate primers and queen genomic DNA templates, with which it was possible to amplify a fragment with mariner transposableelement homology. Phylogenetic analysis demonstrated that this element belongs to the mauritiana subfamily of mariner-like elements and it was named Asmarl. We found that Asmarl is homologous to a transposon described from another ant, Messor bouvieri. The predicted transposase sequence demonstrated that Asmarl has a truncated
\end{abstract}


transposase ORF. This study is part of a molecular characterization of mobile elements in the Atta spp genome. Our finding of mariner-like elements in all castes of this ant could be useful to help understand the dynamics of mariner-like element distribution in the Hymenoptera.

Key words: Transposable element; Mariner-like element; Leaf-cutter ant; Atta sexdens rubropilosa

\section{INTRODUCTION}

Mobile elements are present in all organisms in multiple copies; they have occupied different genomes for millions of years. There is an important division of classes involving these elements, which consider mainly the transposition mechanism. Class I elements transpose via an RNA intermediate, while Class II elements transpose via a cut and paste mechanism.

Class II elements are important tool for genetic transformation in many different organisms. A special transposable element widely used for transformation is the mariner element (Jacobson et al., 1986; Berghammer et al., 1999; Coates et al., 2000; Moreira et al., 2000). This element possesses common characteristics, such as: inverted terminal repeat (ITR) sequence at the extremities; ITR sites flanked by TA nucleotides, duplication of two base pairs in the insertion site (direct repeats); catalytic domain, which contains the D,D(34)D catalytic triad and the transposase domain; DNA-binding domain, which contains the nuclear localization signal and the helix-turn-helix motif, and finally a short size of about $1300 \mathrm{bp}$.

The mariner elements were first identified in Drosophila melanogaster, but are extensively distributed in nature, and can be found in a wide variety of insects and other arthropods. Mariner family members have also been identified in several organisms such as nematodes, marine species, fungi, plants, and mammals, including humans (Robertson, 1993; Capy et al., 1996; Jarvik and Lark, 1998; Leroy et al., 2003; Mandrioli, 2003; Halaimia-Toumi, et al., 2004).

Currently, these elements are members of a large transposon family, known as mariner and mariner-like elements (MLEs) (Lampe et al., 1996). There are over 13 subfamilies known of mariner elements, which typically contain approximately $40-56 \%$ identity in nucleotide and $23-45 \%$ identity in amino acids between the subfamilies and $25-100 \%$ identity in amino acids with a particular subfamily. The phylogenetic history of the mariner element family is known for extensive horizontal transfer between species, some separated by great phylogenetic distances (Robertson and Zumpano, 1997; Robertson and Martos, 1997; Lampe et al., 2003).

Many of these transposable elements belonging to the mariner family are non-functional; they accumulated some kind of mutations during evolution and thus transcribe inactive proteins. The number of MLEs per genome can vary tremendously from species to species (Hartl et al., 1997). Several species that had characterized parcial or full-length elements showed inactive MLEs, with multiple stop codons, deletions or frameshifts. They were observed in the genomes of insects: Rhynchosciara americana (Rezende-Teixeira et al., 2008, 2010), Messor bouvieri (Palomeque et al., 2006), Musca domestica (Yoshiyama et al., 2000), Hessian fly (Russell and Shukle, 1997), Bombyx mori (Robertson and Asplund, 1996; Robertson and Walden, 2003; Kumaresan and Mathavan, 2004), Bactrocera tryoni (Green and Frommer, 2001), Ochlerotatus atropalpus (Zakharkin et al., 2004), in Lepidoptera: Antheraea mylitta (Prasad and Nagaraju, 2003), Mamestra brassicae (Mandrioli, 2003), in nematode: 
Meloidogyne chitwoodi (Leroy et al., 2003), and several other species. The prevalence of inactive copies of mariner elements in many genomes suggests that the vertical inactivation by mutation had an important, probably dominant, role in the evolution dynamic of mariner elements (Lohe et al., 1995).

The present study characterized a transposable element of mariner element family in the leaf-cutter ant genome, Atta sexdens rubropilosa. This insect social behavior and responses to pesticides have been well studied, but there is little data on other aspects of its biology. Recent data comparing the morphology and cytoskeleton organization of the salivary glands (the postpharyngeal, hypopharyngeal, mandibular, and thoracic salivary gland) of different castes show a lack of molecular markers in this system (do Amaral and Machado-Santelli, 2008). It was the starting point for molecular characterization of mobile elements, which nowadays are considered important molecular markers. As observed in many invertebrates the element identified presented defective open reading frame (ORF), stop codons within the ORF and imperfect ITR sequences. However, the study of this element in this system can provide important information on the evolution and function of mobile elements, which always appear to collaborate with a variety of biological functions (revised by Khurana and Theurkaf, 2010; George et al., 2010).

\section{MATERIAL AND METHODS}

\section{Animals}

Ants of A. s. rubropilosa were collected at the Universidade de São Paulo campus, in the Brazilian State of São Paulo, and the wild ants bred in the laboratory.

\section{Nucleic acid}

Genomic DNA was extracted from an A. s. rubropilosa queen ant, added to $300 \mu \mathrm{L}$ TMD (10 mM Tris-Cl, pH 8, $5 \mathrm{mM}$ EDTA, and $0.3 \mathrm{M} \mathrm{NaCl}$ ), and homogenized. This was then mixed with $12 \mu \mathrm{L} 20 \%$ SDS and incubated at $65^{\circ} \mathrm{C}$ for $1 \mathrm{~h}$ in the presence of proteinase $\mathrm{K}(100 \mu \mathrm{g} / \mathrm{mL})$, followed by one extraction with phenol:chloroform. DNA was precipitated in ethanol, washed with $70 \%$ ethanol, dried and dissolved in TE buffer (10 mM Tris-HCl, $\mathrm{pH} 8$, and $1 \mathrm{mM}$ EDTA). DNA was quantified in a NanoDrop ND1000 Spectrophotometer.

\section{PCR, inverse PCR and DNA sequencing}

PCR amplifications were made with degenerated primer to amplify the Asmarl element internal regions (pmar1R: 5'-TTTGCACAACAAGTTCAATTT-3' and pmar1F: 5'-TTTCTGGCAATTTACGGAT-3'). Primers were designed for inverse PCR (iAsmar1R: 5'-TCCAATTAAAGCAGAAAATCAA-3', iAsmar1F: 5'-TGGTGGGACTAAAAGGATCT-3') based on the internal sequences of Asmarl. The protocol of DNA circularization was described in Rezende-Teixeira et al. (2010). The PCR amplifications were performed using Platinum Taq DNA polymerase (Invitrogen Life Technologies) according to manufacturer instructions. Cycle conditions were $94^{\circ} \mathrm{C}$ for $2 \mathrm{~min}, 35$ cycles of $94^{\circ} \mathrm{C}$ for $30 \mathrm{~s} ; 55^{\circ} \mathrm{C}$ for $30 \mathrm{~s} ; 72^{\circ} \mathrm{C}$ for 2 min and a final extension at $72^{\circ} \mathrm{C}$ for $7 \mathrm{~min}$. The PCR products were cloned in pGemT-easy vector (Promega). Clones were sequenced using the BigDye terminator (PerkinElmer) and run 
on an ABI-3100 sequencer (PerkinElmer), using T7 and SP6 primers. The nucleotide sequences were analyzed in a Linux workstation with Phred, Phrap Crosmatch and Consed 17 programs (Ewing and Green, 1998; Ewing et al., 1998; Gordon et al., 1998). The BLAST analyses were done in the non-redundant GenBank database (Wheeler et al., 2000). The MLE sequences of $A$. s. rubropilosa were deposited in the GenBank database and have the following accession No.: A. s. rubropilosa Asmar1 (JF717775).

\section{RESULTS AND DISCUSSION}

To amplify mariner-like transposable elements in the A. s. rubropilosa genome the primers initially used were designed to amplify mariner elements of Rhynchosciara diptera (Rezende-Teixeira et al., 2010). Given the high identity observed between these elements, the primers functioned perfectly, and the ORF internal region of a mariner-like family element was amplified, as expected (pmar1R: 5'-TTTGCACAACAAGTTCAATTT-3' and pmar1F: 5'-TTTCTGGCAATTTACGGAT-3'). To perform all PCRs queen ant DNA of A. s. rubropilosa was used.

Since the ORF internal region was known, a new primer pair was drawn from the amplified sequence of $A$. s. rubropilosa. These primers point to the ends of the transposon to carry out an inverse PCR to provide data of the complete sequence, flanking regions and target site.

The amplified sequence of element in A. s. rubropilosa was named Asmar1, and the consensus element is 1267 nucleotides in length and has the typical structure of an MLE; however, the ITRs are imperfect (Figure 1). An ATG starts at nucleotide 162, one defective ORF of $1035 \mathrm{bp}$ encoding a putative transposase with 345 amino acid protein and 5 internal stop codons. The translation start site, which has been described in some full-length mariner elements, is located in a non-canonical Kozak's box (PuXXATGPu), which was also observed in the element Asmarl of A. s. rubropilosa. The last position of the subject contains a pyrimidine nucleotide (thymidine) in the place of one purine.

The $\mathrm{D}, \mathrm{D}(34) \mathrm{D}$ signature-sequence, which is also a characteristic of mariner elements, defines the second functional domain of the transposase, which is the catalytic domain (129 amino acids in A. s. rubropilosa). This domain is responsible for site-specific cleavage and junction in the transposition process (van Luenen et al., 1994; Craig, 1995). The active site of this domain is defined by three amino acid motifs, consisting of two aspartic acid (D) residues separated by 94 amino acids, followed by another aspartic acid residue separated by 34 amino acids (Robertson, 1993; Doak et al., 1994). The D,D(34)D catalytic triad that makes up the active site serves as a binding domain for the divalent cation $\left(\mathrm{Mg}^{2+}\right)$ required for catalysis (Prasad et al., 2003). The motif D,D(34)D was identified in the Asmarl transposase of $A$. s. rubropilosa with 81 residues, which were $100 \%$ aligned.

It was noted that some sequences (WVPHEL, DEKW, H/QDNAP, HPPYSPDLAPSD), highly conserved in the MLE family (Robertson, 1993; Prasad and Nagaraju, 2003), are also present in the Asmarl element, but some amino acids appear altered. The sequences found in A. s. rubropilosa are: WVPMNL, DEMW, HDNA므, and HPPYSPDVAPSD. The underlined amino acids are shifts found in Asmarl. These sequences correspond to regions of aspartic acid residues that comprise the $\mathrm{D}, \mathrm{D}(34) \mathrm{D}$ catalytic triad and two motifs (WVPHEL and YSPDLAP), typical of mariner transposases described by Robertson (1993).

The ITRs are short sequences of about 28 bp flanking the mobile element, responsible 


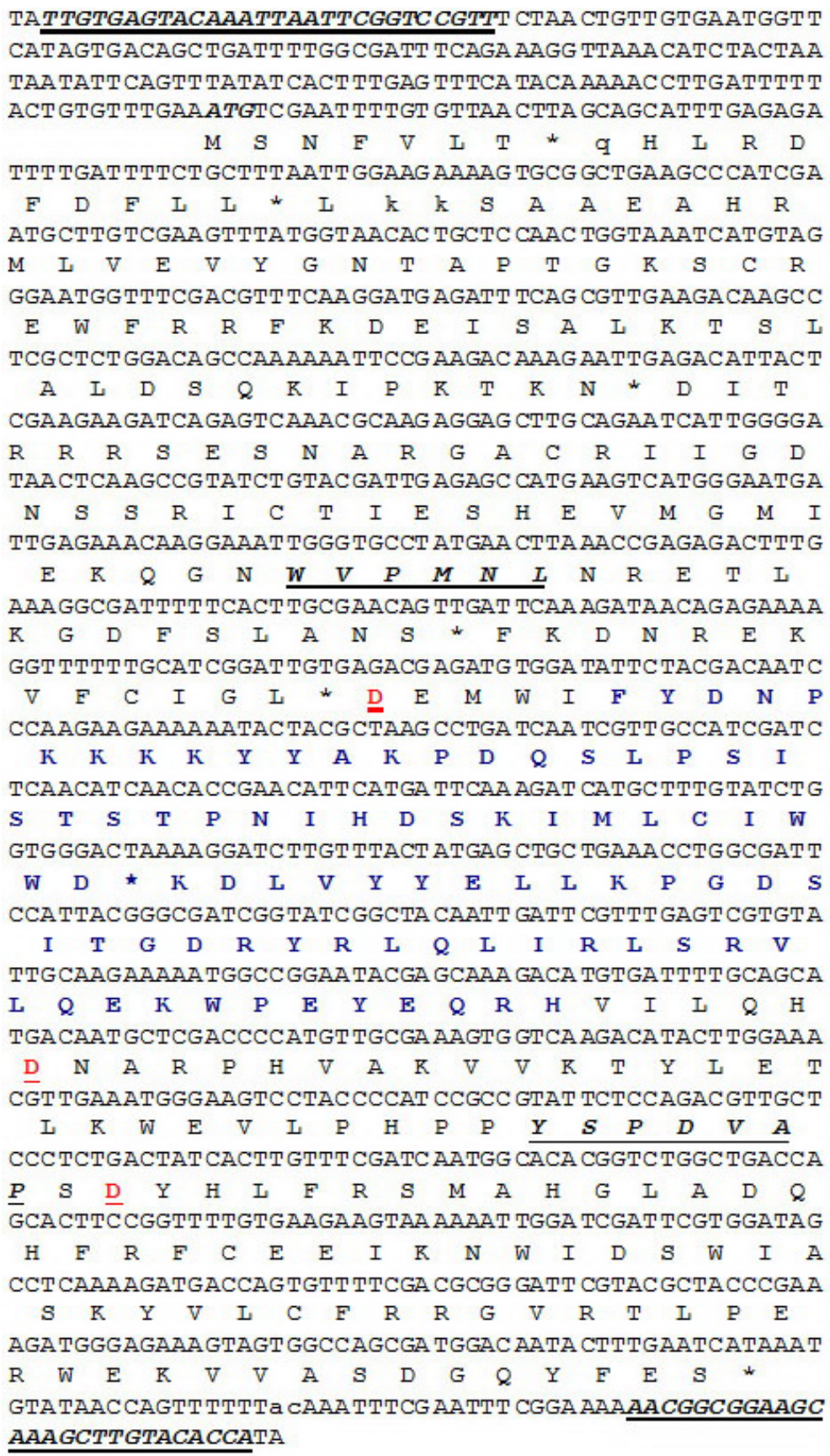

Figure 1. Nucleotide sequence and conceptual translation of the consensus Asmarl element inserted at a duplicated TA dinucleotide site. The inverted terminal repeats (ITRs) and the positions of two conserved motifs described by Robertson (1993) are underlined. The aspartic acids (D) of the D,D(34)D catalytic triad are in red underlined and the catalytic domain in blue. 
for the binding of transposase protein in the transposition mechanism. The binding domain of the ITR sequence of the Mos1 mariner transposase was defined by Augé-Gouillou et al. (2001) analyzing the interaction between the transposase and the ITR sequence. The transposase of MLEs specifically bind as a dimer in the inverted terminal repeat sequence of transposon that encodes it. Two binding motifs were localized in the ITR sequence (Bigot et al., 2005). These motifs are involved in the binding of mariner transposase in ITR. The ITR sequence found for Asmarl is imperfect 5'-TTGTGAGTACAAATTAATTCGGTCCGTT-3'. Thus, in the $A$. s. rubropilosa the transposase is probably non-functional, not only due to stop codons within the ORF, but also imperfect ITR sequences.

A comparison of the terminal repeats showed that there is a high degree of conservation among the sequences of different species. The Asmarl ITR sequence shares extensive identity with the inverted repeats of mauritiana subfamily members (Figure 2). High identity (64\%) is observed between Asmarl from A. s. rubropilosa and Snvmar1 from the fire ant Solenopsis invicta (Krieger and Ross, 2003). This is evidence of the relationship of these mariner elements within A. s. rubropilosa as well as its relationship to mariner elements found in other taxa. Besides, it is possible to observe that Asmarl ITR shares 57-27\% identity with different subfamilies.

\begin{tabular}{|c|c|c|c|}
\hline & $1 \ldots \ldots \ldots 10 \ldots \ldots \ldots 20 \ldots \ldots \ldots 30$ & 8 Identity & Subfami ly \\
\hline Asmar1 & TTGTGAGTACAAATTAATTCGGTCCGTT & -- & \\
\hline Ramar1 & TTGGGTGTACAACTTAATTCCTT CCGTT & 82 & \\
\hline Ramar2 & TTAGGTGTACAAATAAGTTTCTT CCGTT & 72 & mauritiana \\
\hline Sinvmar1 & TTAGGTGTTAAACTTAATTCCTGCCGCT & 64 & \\
\hline $\operatorname{Mos} 1$ & TCAGGTGTACAAGTATGAAATGTCGGTT & 54 & \\
\hline Desmar1 & TTGGGTGTACAACTTAAAAACCGGAATT & 57 & \\
\hline Ammar1 & TTGGGTTGGCAACTAAGTAATTGCGGATTT & 43 & mellifera \\
\hline Bumar1 & CAGGTGTGTAAATATGAAACCGGAATT & 32 & mori \\
\hline Homar1 & TTAGGTCCTTACATATGAAATTAGCGTT & 57 & cecropia \\
\hline Cemar1 & TCAGGTTGTCCCATTTGTTTTTGCACTA & 36 & elegans \\
\hline Himar1 & ACAGGTTGGCTGATAAGTCCCCGGTCTGA & 27 & irritans \\
\hline
\end{tabular}

Figure 2. Comparison and \% identity of inverted terminal repeats (ITR) sequences among Asmarl from Atta sexdens rubropilosa and other elements. The elements represented are Ramarl and Ramar 2 from Rhynchosciara americana, Sinvmarl from Solenopsis invicta, Mos1 from Drosophila mauritiana, Desmarl from Mayetiola destructor, Ammar1 from Apis mellifera, Hcmar1 from Hyalophora cecropia, Cemarl from Caenorhabditis elegans, Himarl from Haematobia irritans, Bmmarl from Bombyx mori.

Figure 3 shows a comparison among ITR sequences of Asmarl, Ramarl and Ramar2. These ITR sequences share a high identity (82-72\%), including six continuous nucleotides (5'-GTACAA) in the 5'-extremity and the six late nucleotides (TCCGTT-3') of the sequence.

However, the presence of an imperfect ITR sequence, generated from mutations may have been the starting point for the inactivation of this element in A. s. rubropilosa. Asmar1, no longer able to undergo transposition, may have accumulated new mutations, creating a totally defective element. Some studies show important characteristics about the ITR sequences, such as conservation of a palindrome sequence and mirror motifs (Bigot et al., 2005), and a pos- 
sible function, initially proposed by Pietrokrovski and Henikoff (1997) and later confirmed by Augé-Gouillou et al. (2001), where the ITR sequence could be a region involved in the mariner transposase binding by HTH motif present in the N-terminal, although the conservation and evolution of ITR sequences in the mariner elements represent a puzzle to be solved.

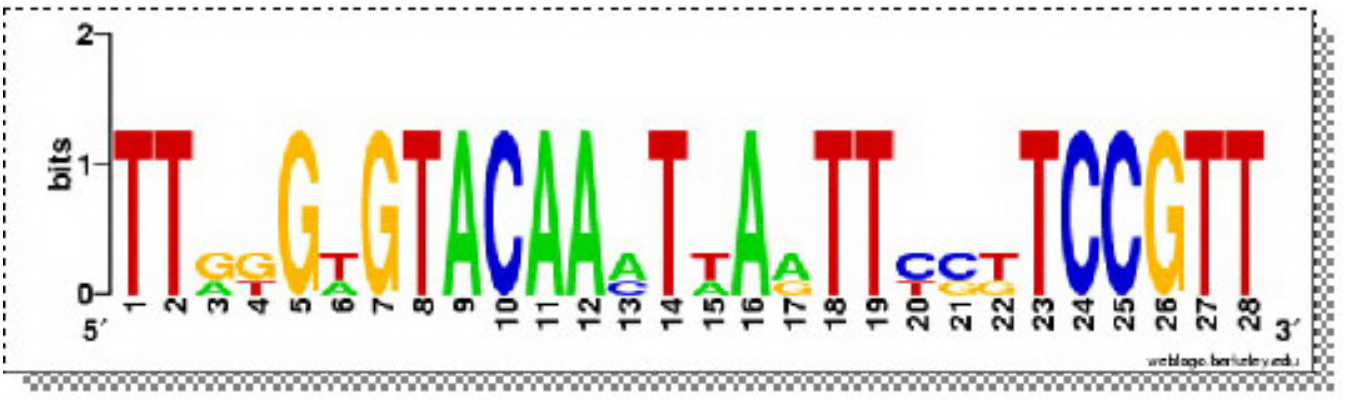

Figure 3. Comparison of inverted terminal repeat (ITR) sequences among Asmarl from Atta sexdens rubropilosa and Ramarl and Ramar 2 from Rhynchosciara americana. The logo was drawn in weblogo.berkeley.edu.

A TA dinucleotide immediately flanks the ITR sequence, as is typical for mariner insertion events. This finding suggests that the Asmarl element has a transposition mechanism similar to other mariner elements. The TA dinucleotide represents the target site of the element, which would then be duplicated upon insertion in the genome.

A phylogenetic tree based on transposase sequences was constructed to compare and investigate the phylogenetic distribution and evolutionary status of the known full-length mariners. The alignment was generated by the use of ClustalX with default parameters and the tree was elaborated by neighbor joining algorithm and constructed with the TreeView 1.6.6 software (Saitou and Nei, 1987; Page, 1996). The protein sequences used were downloaded from GenBank and the accession Nos. are: A. s. rubropilosa Asmarl (JF717775), R. americana Ramar1 (DQ784570), R. americana Ramar2 (DQ784571), D. erecta Demarl (U08094), Apis mellifera Ammarl (U19902), Ceratitis capitata Ccmar1 (AAB17945), Caenorhabditis elegans Cemar1 (NP_497296), C. elegans Cemar2 (NP_497120), Mayetiola destructor Desmar1 (AAA66077), D. mauritiana Mos1 (AAA28678), Haematobia irritans Himar1 (U11645), Chrysoperla plorabunda Cpmar1 (AAA28265), Mantispa pulchella Mpmar1 (U11649), Homo sapiens Hsmar2 (AAC52011), O. atropalpus Atmar1 (AAL16723), Hyalophora cecropia Hcmarl (M63844). A Bmmarl sequence from B. mori (AAB47739) was used as an out-group (Shao and Tu, 2001). The tree obtained was classified into six subfamilies based on their branching pattern (Figure 4). The grouping of Asmarl within the mauritiana subfamily of mariner elements was strongly supported in bootstrap analysis and amino acid identity and similarity with its sister clade.

Figure 5 shows an alignment of Asmarl (Genbank accession No. JF717775) consensus with the Ramarl element of $R$. americana (Genbank accession No. DQ784570), Mboumar element of $M$. bouvieri (Genbank accession No. AJ781769.1) and Desmarl element of $M$. destructor (Genbank accession No. AAA66077). The Asmarl and Mboumar consensus transposase sequences share $44 \%$ amino acid identity and $58 \%$ conservative replacements, while Desmarl transposase shares 45 and $60 \%$ amino acid identity and conservative replacements. 
The Ramarl element appears to be the closest mariner to Asmarl with 46 and $62 \%$ amino acid identity and conservative replacements, respectively.

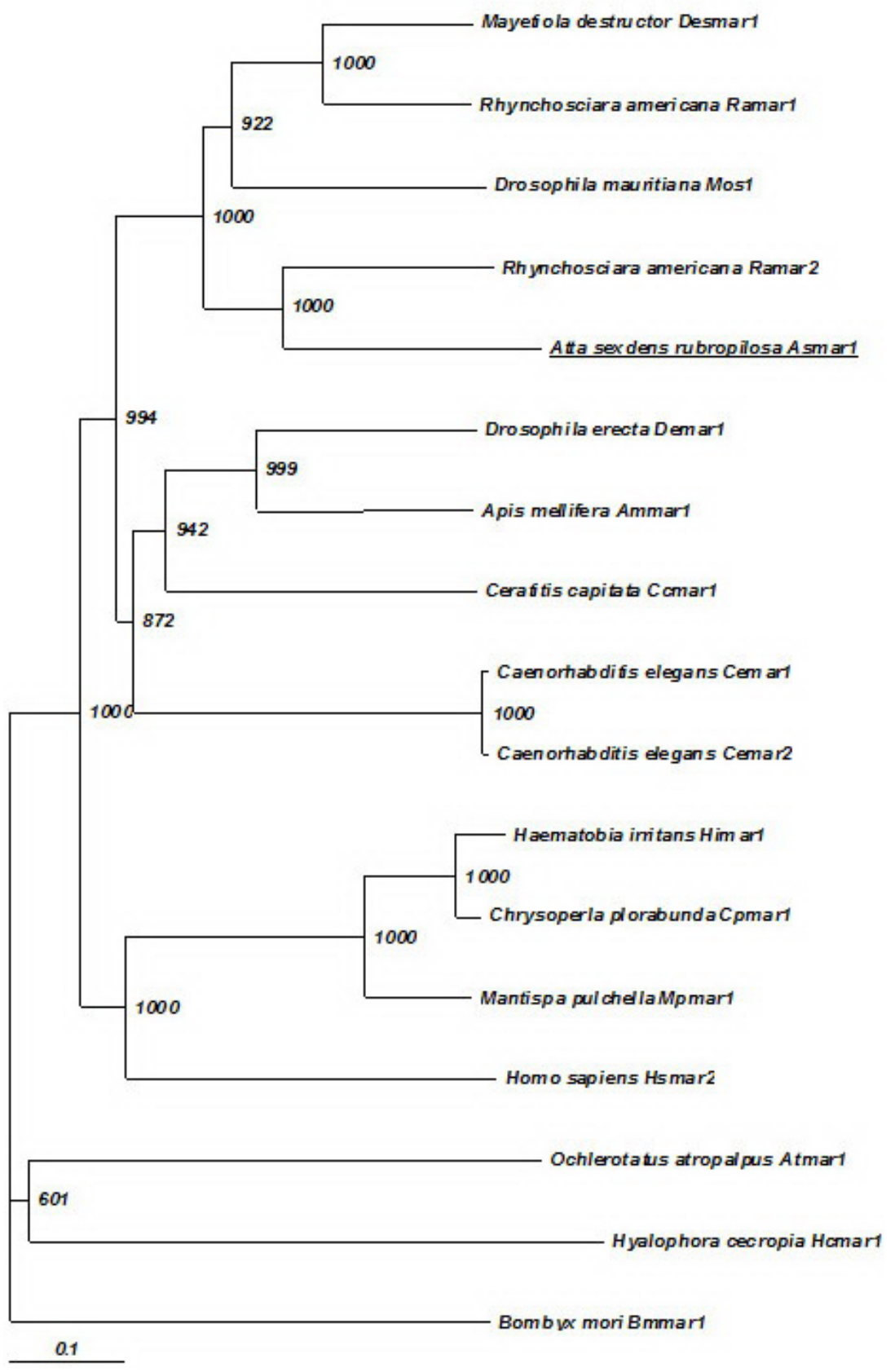

Figure 4. Phylogenetic relationship among the consensus Asmarl and other mariner elements based on their transposase amino acid sequences, using Bmmarl as outgroup. 


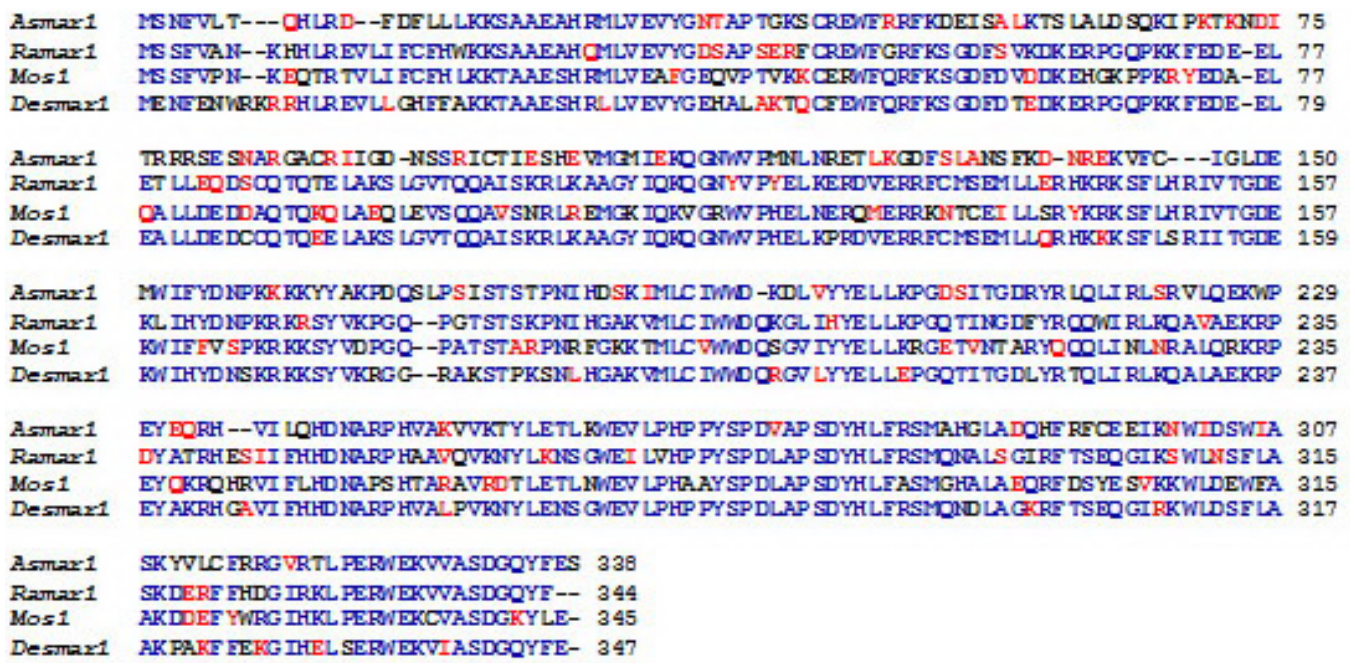

Figure 5. Alignment of the conceptual translation of the consensus Asmarl transposase sequence with those of Rhynchosciara americana Ramar1, Messor bouvieri Mboumar and Mayetiola destructor Desmarl elements.

\section{Concluding remarks}

Leaf-cutter ant colonies of Atta spp are responsible for the largest herbivory impacts in most habitats of the New World tropics (Wilson, 1980). They may account for the destruction of up to $17 \%$ of the total leaf production in tropical rainforests (Begon, 1996). The present study provides the first evidence of a full-length mariner element in the A. s. rubropilosa genome. This transposable element, although non-functional, shows the presence of the mariner element family in the A. s. rubropilosa genome. The high identity among these elements of different model systems led to the amplification of the Asmarl element. Thus, it is possible to relate this fact with the hypothesis of horizontal transfer, which provides that the mariner transposable elements would be inherited from related species, although similarity has already been observed between mariner elements of distant species. These results serve as a step toward understanding the dynamics of MLE distribution in Atta sexdens rubropilosa. Further studies will be important to better characterize the mobile elements in the different castes and to establish its possible association with some relevant cellular function.

\section{ACKNOWLEDGMENTS}

We would like to thank Dr. Paolo M.A. Zanotto, Juliana Velasco and Fernando Lucas Melo for sequencing support and FAPESP and CNPq for their financial support and fellowship.

\section{REFERENCES}

Augé-Gouillou C, Hamelin MH, Demattei MV, Periquet G, et al. (2001). The ITR binding domain of the Mariner Mos-1 transposase. Mol. Genet. Genomics 265: 58-65.

Begon M, Harper JL and Townsend CR (1996). Ecology - Individuals, Populations and Communities. 3rd edn. Blackwell 
Science, Oxford.

Berghammer AJ, Klingler M and Wimmer EA (1999). A universal marker for transgenic insects. Nature 402: 370-371.

Bigot Y, Brillet B and Auge-Gouillou C (2005). Conservation of palindromic and mirror motifs within inverted terminal repeats of mariner-like elements. J. Mol. Biol. 351: 108-116.

Capy P, Vitalis R, Langin T, Higuet D, et al. (1996). Relationships between transposable elements based upon the integrase-transposase domains: is there a common ancestor? J. Mol. Evol. 42: 359-368.

Coates CJ, Jasinskiene N, Morgan D, Tosi LRO, et al. (2000). Purified mariner (Mos l) transposase catalyss the integration of marked elements into the germ-line of yellow fever mosquito, Aedes aegypti. Insect Biochem. Mol. Biol. 30: 1003-1008.

Craig NL (1995). Unity in transposition reactions. Science 270: 253-254.

do Amaral JB and Machado-Santelli GM (2008). Salivary system in leaf-cutting ants (Atta sexdens rubropilosa Forel, 1908) castes: a confocal study. Micron 39: 1222-1227.

Doak TG, Doerder FP, Jahn CL and Herrick G (1994). A proposed superfamily of transposase genes: transposon-like elements in ciliated protozoa and a common "D35E" motif. Proc. Natl. Acad. Sci. U. S. A. 91: 942-946.

Ewing B and Green P (1998). Base-calling of automated sequencer traces using phred. II. Error probabilities. Genome Res. 8: 186-194.

Ewing B, Hillier L, Wendl MC and Green P (1998). Base-calling of automated sequencer traces using phred. I. Accuracy assessment. Genome Res. 8: 175-185.

George JA, Traverse KL, DeBaryshe PG, Kelley KJ, et al. (2010). Evolution of diverse mechanisms for protecting chromosome ends by Drosophila TART telomere retrotransposons. Proc. Natl. Acad. Sci. U. S. A. 107: 21052-21057.

Gordon D, Abajian C and Green P (1998). Consed: a graphical tool for sequence finishing. Genome Res. 8: 195-202.

Green CL and Frommer M (2001). The genome of the Queensland fruit fly Bactrocera tryoni contains multiple representatives of the mariner family of transposable elements. Insect Mol. Biol. 10: 371-386.

Halaimia-Toumi N, Casse N, Demattei MV, Renault S, et al. (2004). The GC-rich transposon Bytmarl from the deep-sea hydrothermal crab, Bythograea thermydron, may encode three transposase isoforms from a single ORF. J. Mol. Evol. 59: 747-760.

Hartl DL, Lohe AR and Lozovskaya ER (1997). Regulation of the transposable element mariner. Genetica 100: 177-184.

Jacobson JW, Medhora MM and Hartl DL (1986). Molecular structure of a somatically unstable transposable element in Drosophila. Proc. Natl. Acad. Sci. U. S. A. 83: 8684-8688.

Jarvik T and Lark KG (1998). Characterization of Soymar1, a mariner element in soybean. Genetics 149: 1569-1574.

Khurana JS and Theurkauf W (2010). piRNAs, transposon silencing, and Drosophila germline development. JBC 191: 905-913.

Krieger MJ and Ross KG (2003). Molecular evolutionary analyses of mariners and other transposable elements in fire ants (Hymenoptera: Formicidae). Insect Mol. Biol. 12: 155-165.

Kumaresan G and Mathavan S (2004). Molecular diversity and phylogenetic analysis of mariner-like transposons in the genome of the silkworm Bombyx mori. Insect Mol. Biol. 13: 259-271.

Lampe DJ, Churchill ME and Robertson HM (1996). A purified mariner transposase is sufficient to mediate transposition in vitro. EMBO J. $15: 5470-5479$.

Lampe DJ, Witherspoon DJ, Soto-Adames FN and Robertson HM (2003). Recent horizontal transfer of Mellifera subfamily Mariner transposons into insect lineages representing four different orders shows that selection acts only during horizontal transfer. Mol. Biol. Evol. 20: 554-562.

Leroy H, Castagnone-Sereno P, Renault S, Auge-Gouillou C, et al. (2003). Characterization of Mcmarl, a mariner-like element with large inverted terminal repeats (ITRs) from the phytoparasitic nematode Meloidogyne chitwoodi. Gene 304: 35-41.

Lohe AR, Moriyama EN, Lidholm DA and Hartl DL (1995). Horizontal transmission, vertical inactivation, and stochastic loss of mariner-like transposable elements. Mol. Biol. Evol. 12: 62-72.

Mandrioli M (2003). Identification and chromosomal localization of mariner-like elements in the cabbage moth Mamestra brassicae (Lepidoptera). Chromosome Res. 11: 319-322.

Moreira LA, Edwards MJ, Adhami F, Jasinskiene N, et al. (2000). Robust gut-specific gene expression in transgenic Aedes aegypti mosquitoes. Proc. Natl. Acad. Sci. U. S. A. 97: 10895-10898.

Page RD (1996). TreeView: an application to display phylogenetic trees on personal computers. Comput. Appl. Biosci. 12: 357-358.

Palomeque T, Antonio CJ, Munoz-Lopez M and Lorite P (2006). Detection of a mariner-like element and a miniature inverted-repeat transposable element (MITE) associated with the heterochromatin from ants of the genus Messor and their possible involvement for satellite DNA evolution. Gene 371: 194-205.

Pietrokovski S and Henikoff S (1997). A helix-turn-helix DNA-binding motif predicted for transposases of DNA transposons. Mol. Gen. Genet. 254: 689-695. 
Prasad MD and Nagaraju J (2003). A comparative phylogenetic analysis of full-length mariner elements isolated from the Indian tasar silkmoth, Antheraea mylitta (Lepidoptera: Saturniidae). J. Biosci. 28: 443-453.

Rezende-Teixeira P, Siviero F, Andrade A, Santelli RV, et al. (2008). Mariner-like elements in Rhynchosciara americana (Sciaridae) genome: molecular and cytological aspects. Genetica 133: 137-145.

Rezende-Teixeira P, Lauand C, Siviero F and Machado-Santelli GM (2010). Normal and defective mariner-like elements in Rhynchosciara species (Sciaridae, Diptera). Genet. Mol. Res. 9: 849-857.

Robertson HM (1993). The mariner transposable element is widespread in insects. Nature 362: 241-245.

Robertson HM and Asplund ML (1996). Bmmar1: a basal lineage of the mariner family of transposable elements in the silkworm moth, Bombyx mori. Insect Biochem. Mol. Biol. 26: 945-954.

Robertson HM and Martos R (1997). Molecular evolution of the second ancient human mariner transposon, Hsmar2, illustrates patterns of neutral evolution in the human genome lineage. Gene 205: 219-228.

Robertson HM and Zumpano KL (1997). Molecular evolution of an ancient mariner transposon, Hsmar1, in the human genome. Gene 205: 203-217.

Robertson HM and Walden KK (2003). Bmmar6, a second mori subfamily mariner transposon from the silkworm moth Bombyx mori. Insect Mol. Biol. 12: 167-171.

Russell VW and Shukle RH (1997). Molecular and cytological analysis of a mariner transposon from Hessian fly. J. Hered. 88: $72-76$.

Saitou N and Nei M (1987). The neighbor-joining method: a new method for reconstructing phylogenetic trees. Mol. Biol. Evol. 4: 406-425.

Shao $\mathrm{H}$ and $\mathrm{Tu} \mathrm{Z}$ (2001). Expanding the diversity of the IS630-Tc1-mariner superfamily: discovery of a unique DD37E transposon and reclassification of the DD37D and DD39D transposons. Genetics 159: 1103-1115.

van Luenen HG, Colloms SD and Plasterk RH (1994). The mechanism of transposition of Tc3 in C. elegans. Cell 79: 293-301.

Wheeler DL, Chappey C, Lash AE, Leipe DD, et al. (2000). Database resources of the National Center for Biotechnology Information. Nucleic Acids Res. 28: 10-14.

Wilson EO (1980). Caste and division of labor in leaf-cutter ants (Hymenoptera: Formicidae: Atta). Behav. Ecol. Sociobiol. 7: $143-156$.

Yoshiyama M, Honda H, Shono T and Kimura K (2000). Survey of mariner-like elements in the housefly, Musca domestica. Genetica 108: 81-86.

Zakharkin SO, Willis RL, Litvinova OV, Jinwal UK, et al. (2004). Identification of two mariner-like elements in the genome of the mosquito Ochlerotatus atropalpus. Insect Biochem. Mol. Biol. 34: 377-386. 